\title{
Biodegradable electrospun PLLA/chitosan membrane as guided tissue regeneration membrane for treating periodontitis
}

\author{
Shuang Chen $\cdot$ Yiting Hao $\cdot$ Wenguo Cui $\cdot$ \\ Jiang Chang • Yue Zhou
}

Received: 7 March 2013/ Accepted: 16 May 2013/Published online: 29 May 2013

(c) The Author(s) 2013. This article is published with open access at Springerlink.com

\begin{abstract}
This paper explores the application potential of a biodegradable PLLA/chitosan electrospun composite membrane for guided periodontal tissue regeneration which in addition serves as a fibroblast barrier. Electrospinning was applied to fabricate the PLLA membrane and aminolysis method was applied to graft chitosan on its surface. The morphology of the PLLA/chitosan membrane was observed by SEM. The surface chemical composition was analyzed by XPS. The appearance of $\mathrm{N}$ $1 s$ peak in XPS demonstrated the successful grafting of chitosan on the PLLA electrospin membrane. After the modification, the water contact angle decreased from $136.9 \pm 2.18^{\circ}$ to $117.0 \pm 2.10^{\circ}$, representing an improved hydrophilicity of the membrane. The bioactivity of the membrane was analyzed by XPS after soaking in SBF. The deposits had a $\mathrm{Ca} / \mathrm{P}$ ratio of 1.6, indicating the hydroxyapatite formation on PLLA/chitosan membrane. The degradation rate was determined by measuring mass loss after immersion in PBS at different time periods. Compared to pure PLLA electrospun membrane which was almost nondegradable, the degradation rate of PLLA/chitosan composite membrane was up to $20 \%$ in 6 weeks while maintaining its basic architecture to keep supporting the
\end{abstract}

S. Chen $\cdot$ Y. Hao $\cdot$ W. Cui $\cdot$ J. Chang $\cdot$ Y. Zhou $(\bowtie)$

Med-X Research Institute, School of Biomedical Engineering,

Shanghai Jiao Tong University, 1954 Hua Shan Road,

Shanghai 200030, People's Republic of China

e-mail: yzhou2009@sjtu.edu.cn

J. Chang $(\bowtie)$

State Key Laboratory of High Performance Ceramics and

Superfine Microstructure, Shanghai Institute of Ceramics,

Chinese Academy of Sciences, 1295 Dingxi Road,

Shanghai 200050, People's Republic of China

e-mail: jchang@mail.sic.ac.cn regenerated tissue. Live-dead cell staining of MC3T3 E1 cells cultured on the surface of the membrane showed a good biocompatibility of the PLLA/chitosan membrane. Furthermore, fibroblast cell line NIH 3T3 was cultured on surface of the membrane for the evaluation of cell penetration. The result demonstrated that the membrane worked as a fibroblast barrier to minimize the unfavorable effect of fibroblasts on periodontal tissue regeneration. Therefore, this electrospun PLLA/chitosan composite membrane has more potential for clinical application compared to old generation regeneration membrane with both suitable degradation rate and non-fibroblast penetration property.

\section{Introduction}

Periodontal diseases are highly prevalent and can affect up to $90 \%$ of the worldwide population. Periodontitis is one of the periodontal diseases leading to loss of connective tissue and bone support, which is a major cause of tooth loss in adults [1]. Severe periodontitis is generally considered to play important roles in connection with several systemic diseases such as coronary artery diseases [2] and diabetes [3-6].

Conventional periodontal treatment have been focused on periodontal tissue regeneration therapy to facilitate periodontal regeneration, such as bone graft [7], guided tissue regeneration (GTR) [8], and stem cell therapy [9], among which the GTR has become the most promising therapy and was widely used in clinical treatment for its convenience and effectiveness [10-14]. GTR treatment of periodontitis is a surgical procedure to implant a regeneration membrane that specifically aims to restore the periodontal tissues supporting the teeth [10]. In this therapy, the ideal regeneration membrane plays a key role to work both as a barrier to prevent 
epithelial and gingival connective cells growing into periodontal defects and a favorable niche to maximize the migration, proliferation of periodontal ligament (PDL) cells, cement oblasts, and osteoblasts within the periodontal defects to promote the reconstruction of the supporting tissue. The regeneration membrane should be biocompatible to integrate with the tissue, allows for space maintenance, and maintains the barrier function for as long as required and easy-to-handle during surgery.

In the history of developing the GTR membrane, nonresorbable materials were the first to be used in clinical treatment, such as the expanded-polytetrafluoroethylene (e-PTFE) [13]. However, materials such as e-PTFE are generally difficult to manipulate and required sutures around the necks of teeth to hold them in place. As the consequence, a second surgical procedure was required to remove them and many complications were reported, especially premature exposure leading to inflammation [15].

To avoid the unfavorable side effects caused by the second surgical procedure, resorbable materials were investigated thus there will be no necessity to retrieve them after the implantation. Nature-derived materials, such as collagen, are generally biocompatible, they degrade too fast before the bone defect and other periodontal tissues fully regenerate [16]. Therefore, composite membranes are better candidates with good biocompatibility and controllable degradation time to meet the requirement of the regeneration period, which is usually at least 4 months. However, good biocompatibility lead to the enhancement of the overall growth rate of epithelial and gingival connective cells, as well as the periodontal cells, which is also an unfavorable side effect.

Electrospinning is a widespread technique for obtaining microfibers and nanofibers, and mesh-like membranes made of those fibers. The fibrous structures produced by this technique present good mechanical properties and a very high specific surface area, which are some of the ideal properties for regeneration membrane of periodontal-guided tissues [17]. Moreover, the surface topology and pore size are adjustable by optimizing the fiber diameters and electrospinning conditions.

Poly (L-lactide) (PLLA) has been safely used in vivo for years. It has been used for three-dimensional cell culture and transplant in dental surgery, which is due to its biodegradability and easy processibility [18]. In Trejo et al. [19] report, PLLA barriers were implanted in intrabony defects of 30 patients after open flap debridement, and all the treatment went out well. However, PLLA is hydrophobic in nature, which makes it hard for cells to attach on the scaffold and limits its applications. Although it is a good candidate for periodontal tissue regeneration, surface modifications for PLLA to improve its hydrophilic and bioresponsivity for cells are necessary.
Many kinds of biomaterial were grafted on PLLA to form a biomimetic interface, among which chitosan was frequently applied. Chitosan has a similar structure with glycosaminoglycan (GAG), which has been proved to be one of the principle components of extracellular matrix (ECM) [20]. Previous result also shows that the chitosan-based scaffolds [21] have excellent biocompatibility and are beneficial of wound healing. Due to its great bioactivity, after the modification of chitosan on PLLA scaffold, the improvement of biocompatibility can be expected.

In this article, a novel composite membrane for the regeneration of periodontal-guided tissues was fabricated using electrospinning of PLLA. The membrane was aminolyzed with chitosan to enhance both the biocompatibility and the degradation [22]. This design allows the regeneration membrane presenting the characteristics of both natural membranes and synthetic materials, such as good biocompatibility and controllable degradation rate. Furthermore, contemporary electrospinning technique endows its unique feature as a fibroblast barrier and demonstrated its great clinical application potential as in periodontitis treatment.

\section{Materials and methods}

Chemicals and cell lines

Poly (L-lactide) was supplied by Jinan Daigang Co. (Jinan, China). Chitosan (low molecular weight) was supplied by Qingdao Medical Institute (Qingdao, China). All other chemicals used in this project were purchased from Guoyao Regents Company (Shanghai, China).

Mouse fibroblast NIH 3T3 cell line was a generous gift from Prof. Hongcheng $\mathrm{Gu}$ of the Nano Biomedical Material Research Center, Med-X Research Institute. The MC3T3 E1 cell line was purchased from the Cell bank of the Chinese Academy of Science (Shanghai, China).

Fabrication of PLLA electrospun membrane

The polymer solution was made by dissolving $1.0 \mathrm{~g}$ PLLA powder in the solvent mixture of $2.5 \mathrm{~g}$ dichloromethane and $1.5 \mathrm{~g}$ acetone. To ensure complete dissolution, the PLLA solution was stirred at room temperature for $4 \mathrm{~h}$. For fabricating the electrospinning membrane, the PLLA solution was drawn into a $2 \mathrm{~mL}$ syringe fitted with a metal syringe needle. A precision pump (LSP01-1A, Baoding Longer Precision Pump Co., Ltd.) was used to supply a steady flow of the solution to the tip of the needle. A highvoltage statitron was used to apply a potential of $25 \mathrm{kV}$ to the syringe needle. A grounded aluminum sheet was placed $12 \mathrm{~cm}$ from the needle tip as the collector. 


\section{Chitosan grafting on PLLA fibers}

The chitosan grafting technique in this paper is based on the previous report by Cui et al. [22] with minor modification. Briefly, to graft amino groups to PLLA electrospun membrane, the membranes were soaked into $0.02 \mathrm{~g} / \mathrm{mL}$ 1,6-hexanediamine/isopropanol solution at $37^{\circ} \mathrm{C}$ for 10 min. Then, the aminolyzed PLLA electrospun membranes were immersed into $2.0 \%$ glutaraldehyde solution. The reaction lasted for $1 \mathrm{~h}$ at room temperature to obtain the aldehyde PLLA electrospun membrane. To graft chitosan on one side of the aldehyde PLLA electrospun membrane, the membranes was incubated in $4 \mathrm{mg} / \mathrm{mL}$ chitosan solution in PBS (phosphate-buffered saline, $\mathrm{pH}$ 7.4) face-down for $24 \mathrm{~h}$ at room temperature. Wash the membrane with water until no free chitosan could be detected in the washing solution. The chitosan-modified PLLA will be designated as PLLA-CS below.

\section{Characterization of PLLA and PLLA-CS membrane}

The surface chemical compositions of PLLA and PLLACS membrane were determined by X-ray photoelectron spectroscopy (XPS, AXIS ULTRA DLD, Kratos Ltd, Britain). PLLA and PLLA-CS samples $\left(20 \times 20 \mathrm{~mm}^{2}\right)$ were directly put on the test stage and total acquisition time was $15 \mathrm{~min}$ for each sample. Overlapping peaks were resolved by the peak synthesis method, applying Gaussian peak components after Shirley-type background subtraction. The surface morphology of pure PLLA and PLLA-CS membranes was observed by a scanning electron microscope (SEM, S-4800, Hitachi). The images of each sample were obtained using the S-4800 cold field emission SEM after $40 \mathrm{~s}$ of gold coating to minimize charging effect. Fiber diameter and pore size were characterized quantitatively by image analysis of SEM images. Images were imported into Nano Measurer (download from the internet, edition 1.2.5), and the fiber diameters of at least 50 fibers per image were measured to get the average diameter. To measure the pore size of membranes, the diameter of a virtual sphere between fibers in the same or in the nearby plane was measured; at least 20 spheres were chosen randomly per image. Water contact angle of PLLA and PLLACS was measured at room temperature to evaluate surface wettability of the membranes. The water contact angles were measured with a Kruss GmbH DSA 100 Mk 2 goniometer (Hamburg, Germany) followed by image processing of sessile drop with DSA 1.8 software.

\section{Material degradation}

PLLA and PLLA-CS membranes were put in $20 \mathrm{ml}$ PBS and incubated at $37{ }^{\circ} \mathrm{C}$. The initial mass of each sample was around $15 \mathrm{mg}$; each sample group was tested in triplicates. The PBS was changed every week. After 2, 4, and 6 weeks, the membranes were rinsed with plenty of water and dried for $24 \mathrm{~h}$ using freeze-drying method.

\section{Mineralization process}

The PLLA-CS membranes and pure PLLA membranes were immersed in $20 \mathrm{~mL}$ simulated body fluid (SBF) at $37^{\circ} \mathrm{C}$ for 9 days. Fresh SBF solution was changed every other day. After this process, the membranes were rinsed gently with pure water and dried using freeze-drying method for $24 \mathrm{~h}$. The mineralized PLLA-CS membrane with the deposition of HA would be designated as PLLACS-HA below.

\section{Bioactivity of PLLA-CS}

To investigate the bioactivity of PLLA-CS, the formation of hydroxyapatite (HA) on the surface of PLLA-CS membrane was evaluated by XPS. Details of XPS procedures have been elaborated above.

\section{Cell culture}

The membranes were cut into round shapes with the diameter of $15 \mathrm{~mm}$ to fit right into the wells of a 24-well plate (Corning, NY). Titanium rings were used to immobilize the membranes to prevent the membrane from floating during the experiment. NIH 3T3 and MC3T3 E1 were seeded on the membrane with the density of $2 \times 10^{4} /$ well. The culture medium for NIH 3T3 was DMEM and $\alpha$-MEM for MC3T3 E1 (Gibco BRL, Rockville, MD). Both media were supplemented with $10 \%$ fetal bovine serum (FBS, Hiclone, Thermo Fisher Scientific Inc., Beijing, China) and $1 \%$ penicillin and streptomycin (Gibco, Invitrogen, USA). The cell culture media were changed every 2 days.

\section{Cell proliferation and compatibility}

Cell proliferation was evaluated by counting the number of DAPI-stained cells under a fluorescence microscope. In brief, after MC3T3 E1 cells were cultured for 1, 3, and 7 days, the membranes with the cells were moved from the culture medium, washed with PBS, and stained by DAPI ( $5 \mu \mathrm{g} / \mathrm{mL}$, Beyotime Institute of Biotechnology, China) for $5 \mathrm{~min}$ in room temperature. The cells were visualized under a fluorescence microscope at $364 \mathrm{~nm}$ exciting wavelength. For each sample, pictures were taken from 10 randomly selected visual fields and the total number of cells was determined by Image $\mathbf{J}$ (http://rsbweb.nih.gov/ij/, version $1.42 q)$. 
Live-dead cell staining (LIVE/DEAD Viability/Cytotoxicity Kit, Molecular Probes Inc., USA) was used to evaluate the biocompatibility of the membrane. After MC3T3 E1 cells were cultured for 1, 3, and 7 days, the membranes with the cells were removed from the culture medium and washed twice with D-PBS (Dulbecco's phosphate-buffered saline). Calcein AM and EthD-1 were diluted to 2 and $4 \mu \mathrm{M}$ by D-PBS for staining, and the reaction was incubated in $37{ }^{\circ} \mathrm{C}$ for $15 \mathrm{~min}$. The dead cells were visualized under $530 \mathrm{~nm}$ exciting wavelength, and the live ones were visualized under $485 \mathrm{~nm}$ exciting wavelength under a fluorescence microscopy (DMI3000B, Leica, Germany). The images obtained were processed using the Leica application suite (Leica Microsystems, Wetzlar, Germany). Image $\mathbf{J}$ was used to merge the fluorescence microscope images. For each sample, pictures were taken from 3 randomly selected visual fields and the total numbers of both living and dead cells were calculated by cell count tool of Image $\mathrm{J}$.

Cell penetration

NIH 3T3 was seeded on one side of the PLLA-CS membrane without the grafting of chitosan. After 5 days, the membrane was removed form cell culture medium and washed twice with PBS. Slit the membrane in the width of $50 \mu \mathrm{m}$ by freezing microtome (CM1900, Leica, Germany). After DAPI staining, the membrane slice was observed by fluorescence microscope (DMI3000B, Leica, Germany).

\section{Statistical analysis}

The values were expressed as means (standard deviation) (SD). Whenever appropriate, two-tailed Student's $t$ test was used to discern the statistical difference between groups. A probability value $(P)$ of $<0.05$ was considered to be statistically significant.

\section{Results and discussion}

\section{Characterization of PLLA-CS membrane}

The chemical composition of PLLA and PLLA-CS were determined by XPS. Figure 1a shows the appearance of $\mathrm{N}$ $1 s(400.1 \mathrm{eV})$ signal of PLLA-CS. The resolved $\mathrm{N}$ $1 s$ spectra of PLLA-CS membranes are shown in Fig. $1 \mathrm{~b}$. The magnified $\mathrm{N} 1 s$ peaks of XPS spectra were presented in gray color in the background. Apparently two peaks could be identified. The peak at $399.8 \mathrm{eV}$ was the overlapping peaks of both $\mathrm{N}-\mathrm{C}=\mathrm{O}$ and $\mathrm{NH}_{2}$ groups in chitosan [23], while the peak at $401.6 \mathrm{eV}$ was assigned to amino groups in the ammonium form $\left(-\mathrm{NH}_{3}{ }^{+}\right)$[24]. Both forms of Nitrogen were likely to present in chitosan, taking into account the pKa of chitosan amine groups and the fact that chitosan was dissolved in PBS before being grafted on PLLA electrospun fibers. In addition, in Table 2, the quantified $\mathrm{N}$ atom concentration at $400.1 \mathrm{eV}$ of PLLA-CS membrane was $1.76 \%$ while pure PLLA membrane had none. These results indicated that grafting of chitosan on PLLA was successful.

The SEM images revealed uniform sizes of diameter and smooth surfaces for both PLLA and PLLA-CS fibers (Fig. 2). This result showed that chitosan modification of PLLA scaffold did not change the morphology of the surface of the fiber. The average diameter of pure PLLA fiber was $1.06 \pm 0.13 \mu \mathrm{m}$, while that of PLLA-CS fiber was $1.15 \pm 0.18 \mu \mathrm{m}$. Statistical analysis showed no significant difference between these two kinds of fibers in diameter. The average pore size of PLLA scaffold was $3.18 \pm 0.57 \mu \mathrm{m}$, and that of the modified scaffold PLLACS was $2.76 \pm 0.72 \mu \mathrm{m}$ under our experimental condition. There was no significant difference between pore sizes either.

The water sessile drops on PLLA and PLLA-CS are shown in Fig. 3. The water contact angle of PLLA electrospun membrane was $136.9 \pm 2.18^{\circ}$ while that of
Fig. 1 The surface chemical composition of PLLA and PLLA-CS. a N $1 s$ peaks of XPS spectra of PLLA and PLLA-CS, b the resolved narrow-scan spectra of $\mathrm{N} 1 s$ region of PLLA-CS
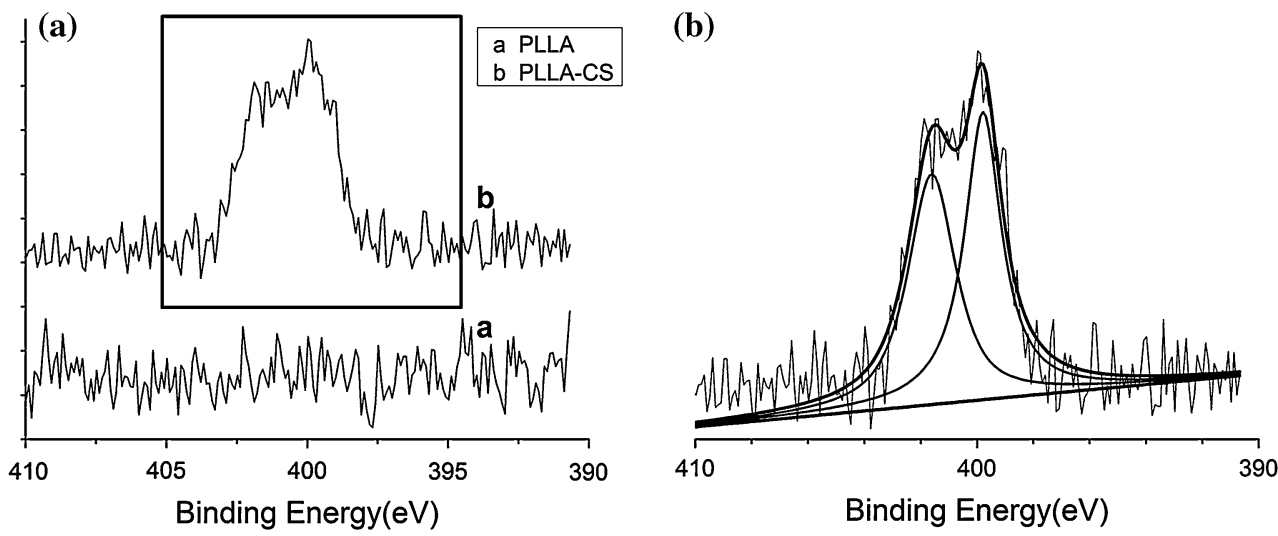

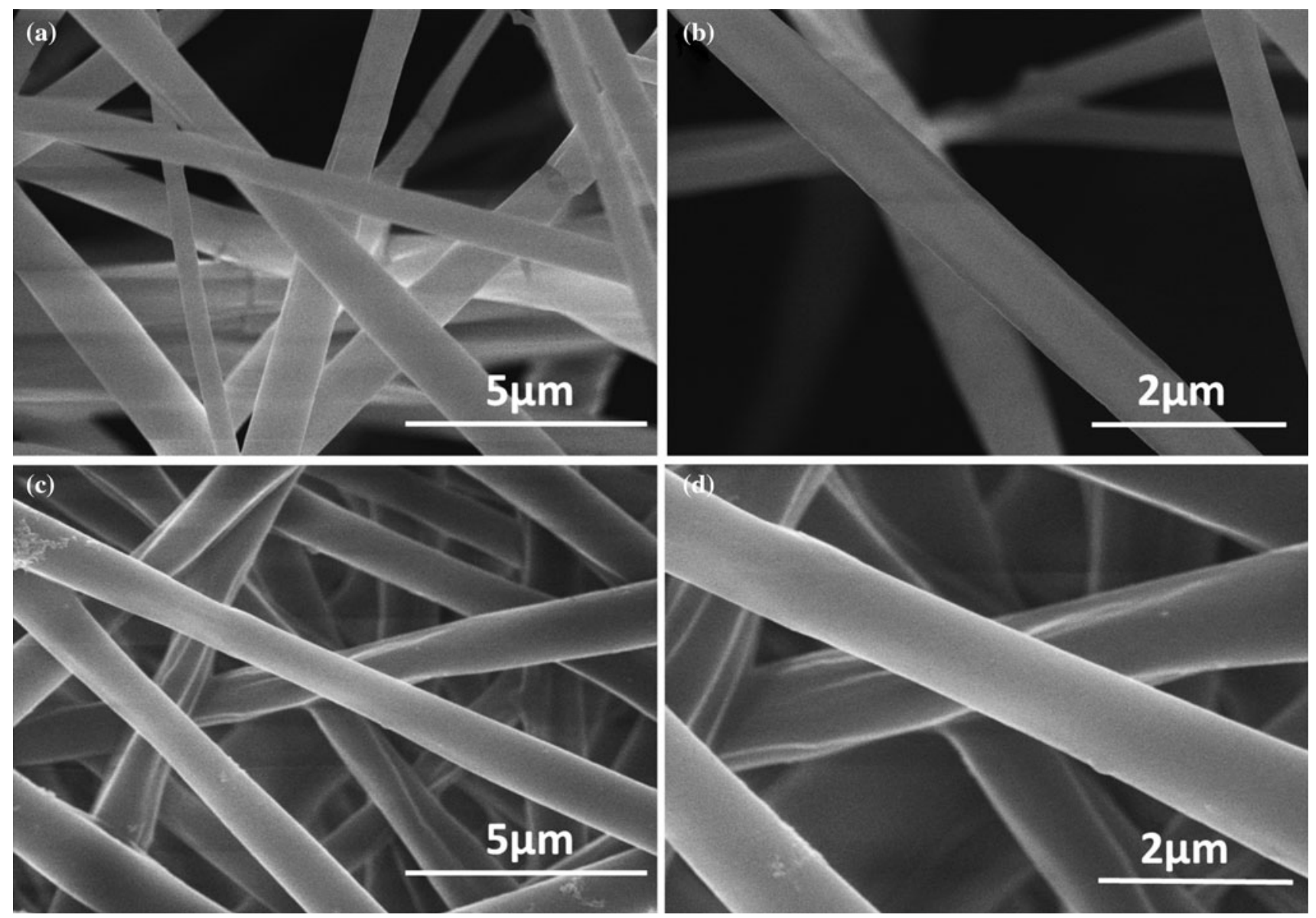

Fig. 2 The surface morphology of PLLA and PLLA-CS fibers. SEM images of pure electrospun PLLA membrane: $\mathbf{a} \times 10,000, \mathbf{b} \times 20,000$; SEM images of PLLA-CS, the chitosan-grafted membrane, $\mathbf{c} \times 10,000, \mathbf{d} \times 20,000$

PLLA-CS was $117.0 \pm 2.10$ (Table 1). The modification of chitosan on the surface significantly decreased the water contact angle of the chitosan-grafted membranes, representing an improved hydrophilicity.

PLLA has been widely applied due to its excellent biodegradability and nontoxicity of the degradation products. However, poor hydrophilicity limit the utility of PLLA [25]. Chitosan grafting is a common way to improve the properties of material such as bacteriostatic effect [26], enhancing adsorption properties [27], and improving biocompatibility [28]. In our research, chitosan helped to promote hydrophilicity of the PLLA membrane. SEM result showed that the surface roughnesses of PLLA membranes were almost consistent before and after the modification of chitosan. However, the water contact angle of PLLA electrospun fibers scaffold decreased from 136.9 to 117.0 after the modification, according to Fig. 3 and Table 1, which is attributed to the hydrophilicity of chitosan.

There are several ways to graft chitosan on PLLA scaffold. In our research, aminolysis method was selected due to the stability of product and easy operability. The successful modification process contains three steps, according to a previous research [22]. As shown in the scheme (Fig. 4), the amino group is introduced on PLLA in the first step. In this step, the ester group or carboxyl group $(-\mathrm{COOH})$ on the surface of the PLLA fibers reacts with one amino group $\left(-\mathrm{NH}_{2}\right)$ in 1,6-hexanediamine, which will result in one free animo group on the chain of the aminolyzed PLLA polymers. The second step is to turn the amino groups into aldehyde groups $(-\mathrm{CHO})$. By the reaction of glutaraldehyde and $-\mathrm{NH}_{2}$, free aldehyde groups are successfully grafted. Finally, chitosan can be binded with PLLA through the aldimine condensation since there are plenty amino groups on chitosan. Since the amount of chitosan grafted on scaffolds was only determined by amino groups, it can be controlled easily by changing experimental conditions. Moreover, this method allows the amino groups on the chitosan for further modifications by methods of covalent bonding [29] and layer-by-layer assembly [30].

\section{Bioactivity of PLLA-CS}

XPS was used to examine the surface chemical composition of PLLA-CS after the immersion in SBF. Table 2 shows the 
Fig. 3 Hydrophilicity of PLLA and PLLA-CS. The images of water sessile drop on a PLLA and b PLLA-CS
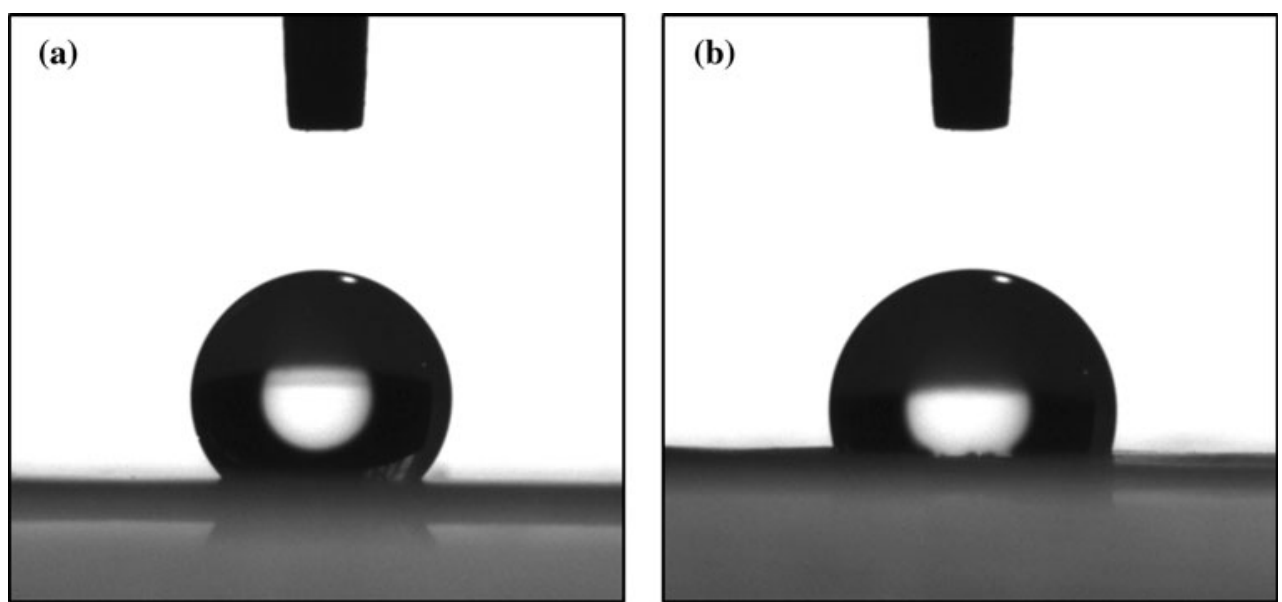

Table 1 Water contact angles of PLLA and PLLA-CS membrane

\begin{tabular}{ll}
\hline Sample & Water contact angles $\left(^{\circ}\right)$ \\
\hline PLLA & $136.9 \pm 2.18$ \\
PLLA-CS & $117.0 \pm 2.10$ \\
\hline
\end{tabular}

$\mathrm{X}$-ray photoelectron spectroscopy quantitative analysis result of all the samples. Only in PLLA-CS-HA, Ca $2 p(347.1 \mathrm{eV})$ and $\mathrm{P} 2 p(132.9 \mathrm{eV})$ signals were observed. The ratio of $\mathrm{Ca} / \mathrm{P}$ in PLLA-CS-HA was 1.6 , which was corresponding to the stoichiometric ratio (1.67) of HA $\left(\mathrm{Ca}_{10}\left(\mathrm{PO}_{4}\right)_{6}(\mathrm{OH})_{2}\right)$. The XPS data proved the formation of HA on the surface of chitosan-grafted PLLA fibers, indicating that the PLLA-CS membrane has bioactivity.

The formation of HA on the surface of a biomaterial is important for the materials to bond with the surrounding tissue [31, 32] after implantation. The biomineralization on PLLA-CS membrane is relevant to the special ionic groups and polar groups of chitosan. It is generally considered that heterogeneous nucleation is the causation of mineral crystalline during tissue calcification [33]. The negatively charged $-\mathrm{OH} / \mathrm{C}=\mathrm{O}$ groups and the positively charged amino groups of chitosan provided the nucleation sites for $\mathrm{HA}$ crystalline through binding or chelating the $\mathrm{Ca}^{2+}$ and $\mathrm{PO}_{4}{ }^{3-} / \mathrm{OH}^{-}$ions with positive and negative charges, respectively [34, 35]. Therefore, the modification of chitosan will accelerate the enrichment of the $\mathrm{Ca}^{2+}$ ions on $-\mathrm{OH}$ and $\mathrm{C}=\mathrm{O}$ groups of chitosan, while the anions (such as $\mathrm{PO}_{4}{ }^{3-}$ and $\mathrm{OH}^{-}$) will assemble on the amino groups and the calcium complexes due to electrostatic interaction and polar interaction after soaking in SBF [36]. In other word, the enrichment of ions like $\mathrm{Ca}^{2+}, \mathrm{PO}_{4}{ }^{3-}$, and $\mathrm{OH}^{-}$triggers the heterogenous nucleation for mineralization and induces the deposition of HA. With the increase of soaking time, more HA crystalines deposited, and ultimately the inorganic HA layer was achieved.
Degradation

After immersed in PBS, the PLLA-CS membrane began to degrade, and the change of morphology surface started to be able to be observed after 14 days (Fig. 5a). The surface became rougher when the immersing time was longer (Fig. 5a-d). However, up to 6 weeks, the membrane managed to maintain its basic architecture (Fig. 5d). On the contrary, the pure PLLA membrane degraded quite slowly in PBS, losing only $5 \%$ of its original weight after 6 weeks, while PLLA-CS membrane lost more than $20 \%$ of its original weight (Fig. 5e). The result showed that the degradation rate of PLLA scaffold increased significantly after chitosan grafting.

PLLA is an aliphatic polyester, which degrades mainly through hydrolysis of the hydrolytically unstable ester linkage in the polymer's backbone. Therefore, a decrease of molecular weight can be observed before any physical mass loss during the degradation [37]. When the molecular weight of the polymer decreases to a critical value after degradation, the soluble oligomers will be released to effect the mass loss.

It was observed in our research that the degradation rate of PLLA scaffold increased significantly after chitosan grafting. This may due to the following reasons. First, imine groups $(-\mathrm{CH}=\mathrm{N}-)$ were introduced on PLLA fibers through the modification. The hydrolysis of imine groups as well as ester groups led to the degradation of PLLA-CS which resulted in extra mass loss, while the PLLA degradation was mainly caused by the hydrolysis of ester groups [38]. Second, during the aminolysis process, the alkalinecatalyzed degradation of polylactide acid matrix was observed, which resulted in a decrease of molecular weight even at rather short reaction time and destroyed the fibrous structure [39]. In our study, 1,6-hexanediamine was used for the reaction between the ester groups of PLLA and diamine. The reaction broke the ester linkage in the 
Fig. 4 Reaction scheme of chitosan grafting. Schematic drawing of surface modification procedures of electrospun PLLA fibers (a)

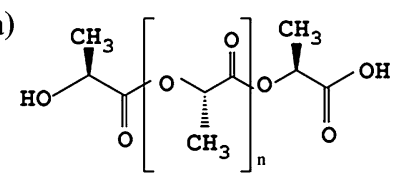

PLLA

(d)

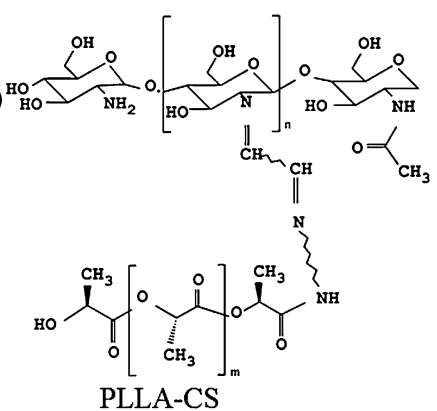

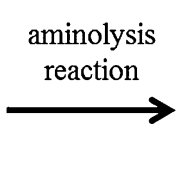

(b)<smiles>CC(=O)C(C)OC(C)C(=O)OC(C)C(=O)NCCCN</smiles>
aminolyzed PLLA

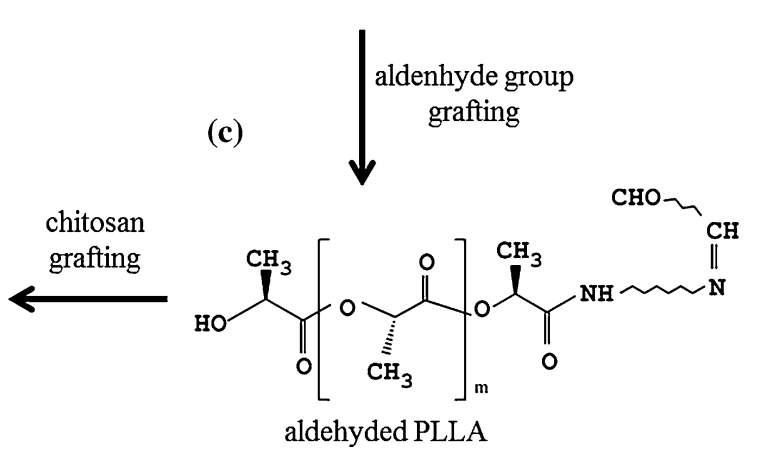

Table 2 X-ray photoelectron spectroscopy quantitative analysis of the as-prepared samples: relative chemical compositions (at.\%) of PLLA, PLLA-CS, and PLLA-CS-HA

\begin{tabular}{|c|c|c|c|c|c|c|}
\hline & \multicolumn{5}{|c|}{ Binging energy $(\mathrm{eV})$ of XPS peaks } & \multirow[b]{2}{*}{$\mathrm{Ca} / \mathrm{P}$} \\
\hline & $\mathrm{C} 1 s$ & $\mathrm{O} 1 s$ & $\mathrm{~N} 1 s$ & $\mathrm{Ca} 2 p$ & $\mathrm{P} 2 p$ & \\
\hline Sample & 284.8 & 532.0 & 400.1 & 347.1 & 132.9 & \\
\hline PLLA & 64.33 & 35.67 & - & - & - & - \\
\hline PLLA-CS & 61.72 & 35.53 & 1.76 & - & - & - \\
\hline PLLA-CS-HA & 62.49 & 35.26 & 1.64 & 0.37 & 0.24 & 1.6 \\
\hline
\end{tabular}

polymer's backbone which also contributed to the relatively higher degradation rate of PLLA-CS. Moreover, the high voltage of the electrospinning process resulted in the enrichment of methyl groups on the surface of electrospun PLLA fibers. Therefore, the PLLA electrospun membrane had a hydrophobic surface which was preventative for water attack of the fiber [40]. However, the modification of chitosan improved the hydrophilicity of PLLA membrane and made it easier for water to get into the fiber. The water molecule hydrolyzed the amorphous areas, which accelerated the crystalline parts of PLLA dropping off from the fibers and further led to the increase of the mass loss [41].

To avoid the secondary surgery on the patient to retrieve the scaffold, it is reasonable to design a scaffold with the degradation rate both satisfying the time of tissue regeneration and degrade after that without the necessity of surgery. Since the average periodontal tissue regeneration time is around 6-8 weeks, in our experiment, the amount of the chitosan grafted on PLLA membrane was optimized to meet that requirement. In 6 weeks, the degradation is up to $20 \%$ but the PLLA-CS membrane still maintained its basic architecture to keep supporting the regenerated tissue. Therefore, this biodegradable PLLA-CS composite membrane has greater advantages compared to current commercially available GTRs [42] in clinical application.

Cell compatibility and proliferation on PLLA-CS membrane

Figure $6 a-i$ show the live-dead cell staining result of MC3T3 E1 after being cultured for 3, 5, and 7 days onto the surface of the three kinds of membranes: PLLA, PLLACS, and PLLA-CS-HA. The living MC3T3 E1 cells were stained green and exhibited their normal shape when adhered to a surface. On the contrary, the dead ones were round-shaped and stained in red color. As shown in Fig. 6j, the percentage of living cells of PLLA-CS membrane is significantly higher than that of PLLA at day 3, day 5 and day 7. Furthermore, the same comparison of the density and percentage of living cells on PLLA-CS-HA was higher than that of PLLA-CS. The result demonstrated that both modification and mineralization improved the cell adhesion and cell compatibility of the PLLA membrane significantly.

The MC3T3 E1 cells were cultured on PLLA, PLLACS, and PLLA-CS-HA membranes for 1, 3, and 7 days. At the end of each culture period, the membrane was stained with DAPI and the number of total cells was calculated. During the whole cell culture period, the cell number of PLLA and PLLA-CS did not change significantly. However, compared with day 1 , cell number of PLLA-CS-HA showed a significant increase after 3 and 7 days (Fig. 7). Moreover, after 3 and 7 days, cells grown significantly faster on PLLA-CS-HA membrane than both pure PLLA and PLLA-CS. As it is showed in the figure, after 7 days, the cells on PLLA-CS-HA was almost twice as much as these on the other two kinds of membranes.

The poor biocompatibility of PLLA may be due to the lack of natural recognition sites on polyester surfaces for 

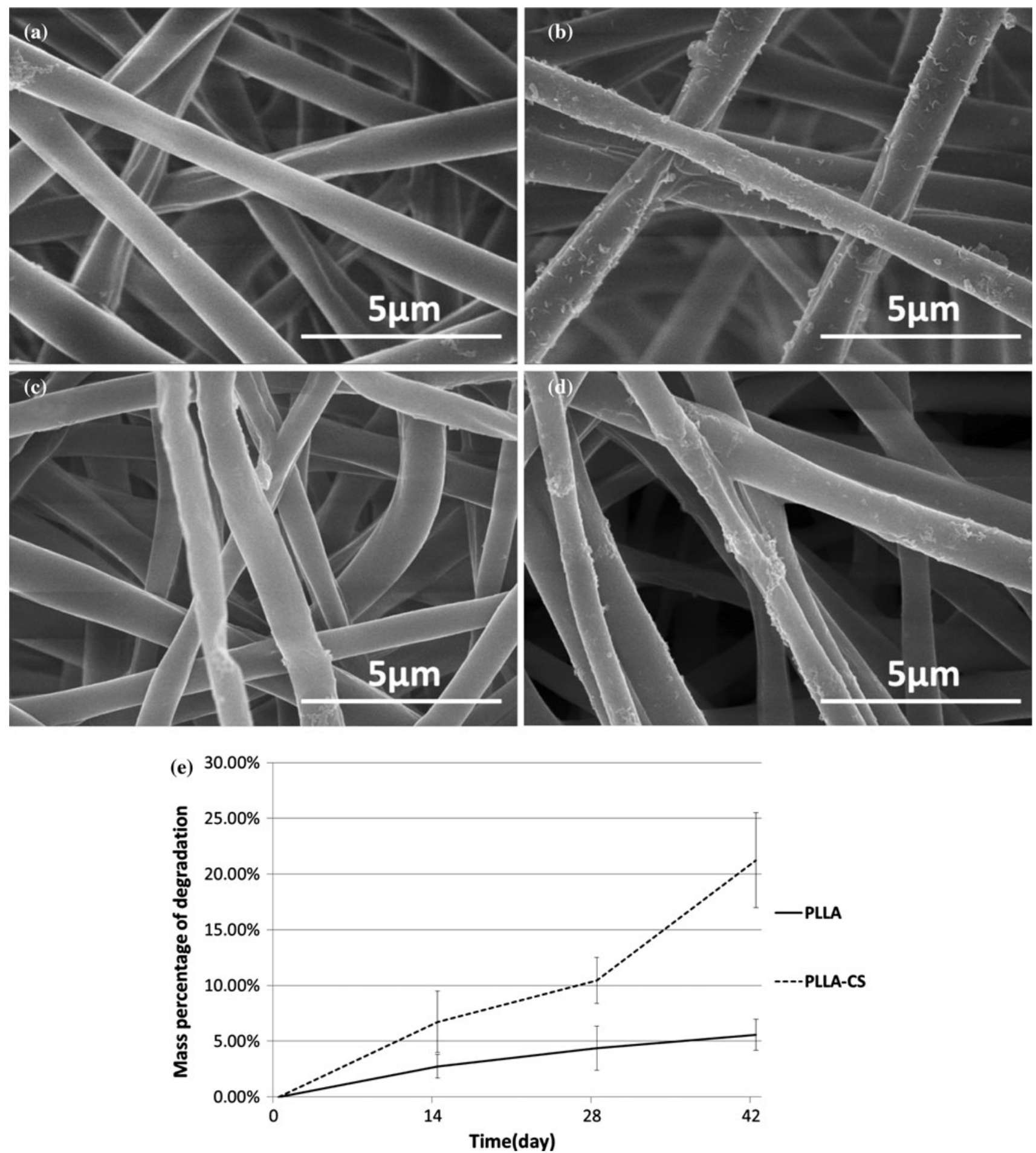

Fig. 5 In vitro degradation. The degradation results of PLLA and PLLA-CS membrane in vitro. The SEM images $(\times 10,000)$ of a original PLLA-CS membrane, PLLA-CS membrane after

covalent cell recognition signal molecules [43]. Polysaccharides outside the cell membrane are very important signal molecules. Chitosan can mimic ECM and act as polysaccharides to help cells to adjust their growth and b 2 weeks, c 4 weeks, and d 6 weeks' degradation in PBS, e the mass loss curve of PLLA, PLLA-CS in PBS

function [44]. Besides, chitosan is a very good cation absorber [45] and the membrane acts as a positively charged center in solution. The electronic interaction makes it easier for the artificial scaffold to bind with 

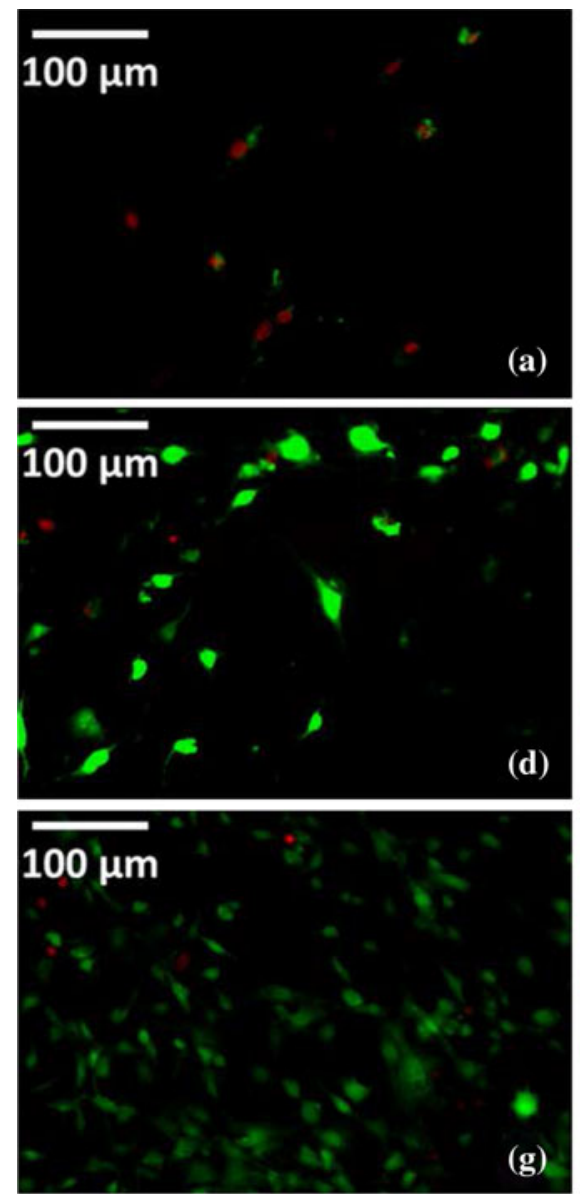
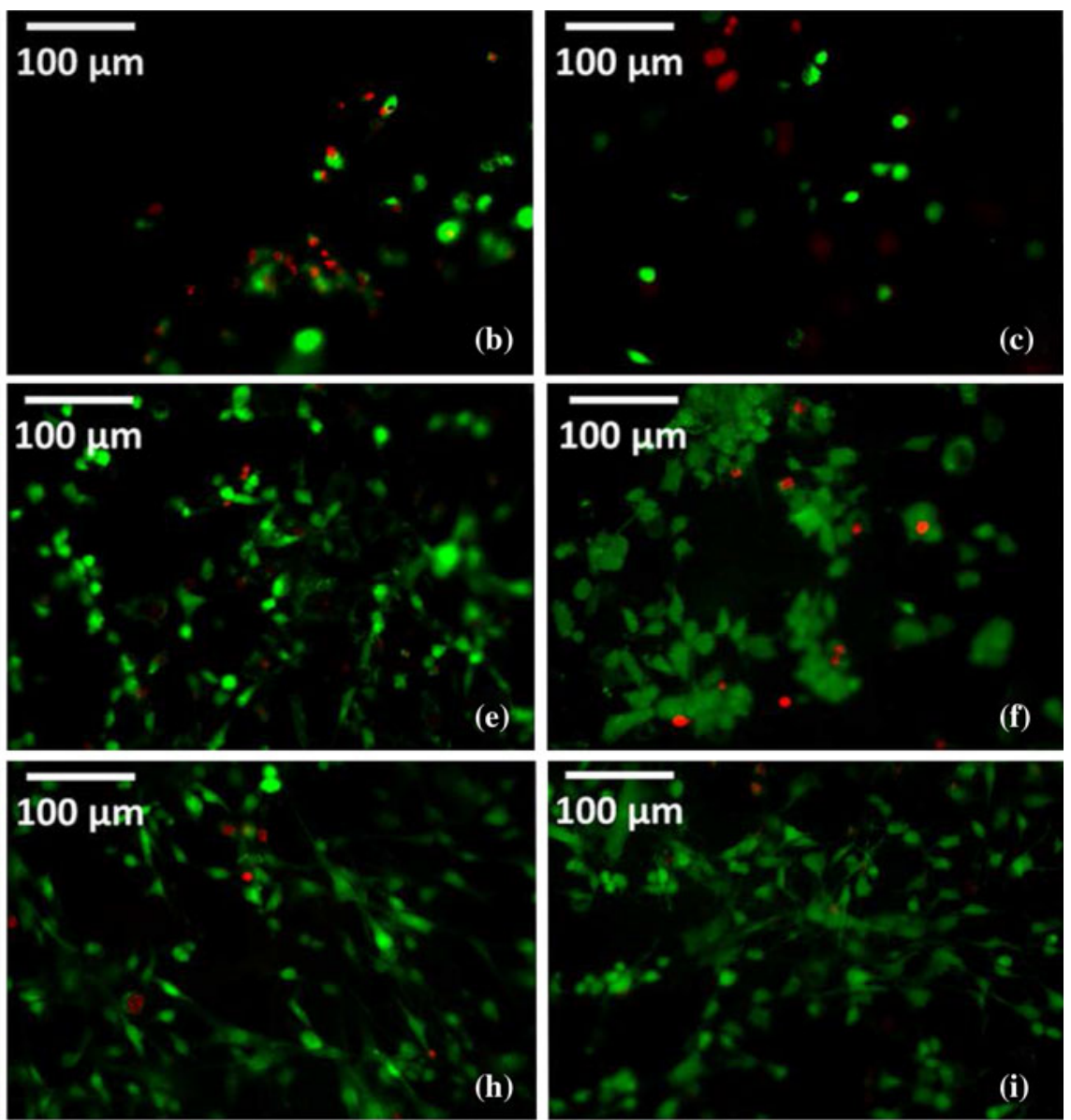

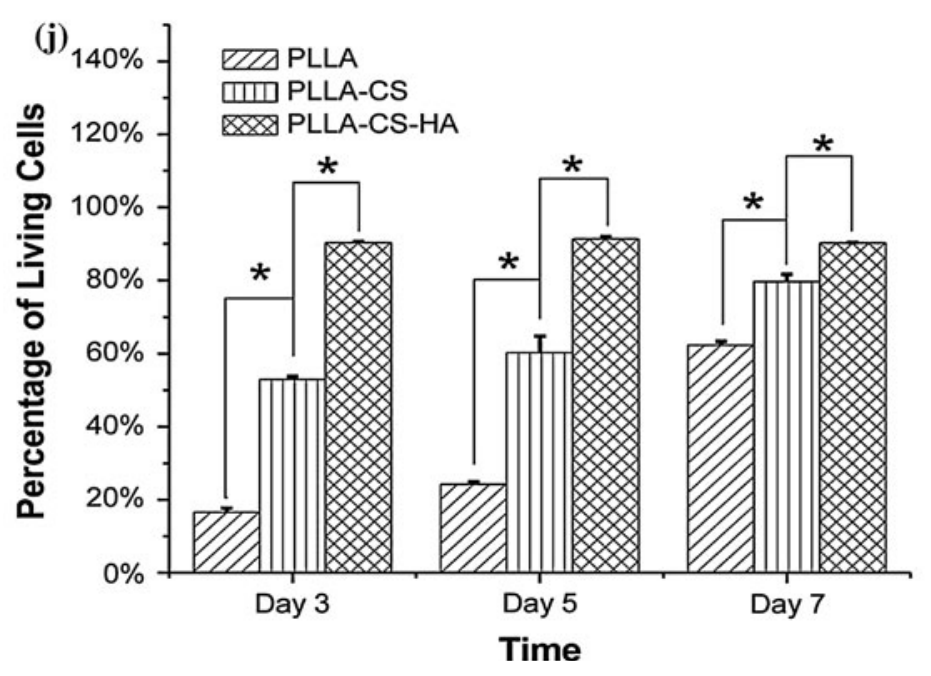

Fig. 6 Cell compatibility. Fluorescence microscope images of MC3T3 E1 cells after Live-dead staining. MC3T3 E1 cells were cultured on a-c PLLA, d-f PLLA-CS, and g-i PLLA-CS-HA for a, d,

$\mathbf{g} 3$ days, $\mathbf{b}, \mathbf{e}, \mathbf{h} 5$ days, $\mathbf{c}, \mathbf{f}, \mathbf{i} 7$ days, $\mathbf{j}$ the percentage of living cells after MC3T3 E1 cells cultured on 3 kinds of membranes $(* P<0.05$, $n=3$ )

anchoring proteins and helps cells attach to the surface. In addition, with the deposit of HA, the surface roughness of PLLA-CS membrane [46] increases, which may also contribute to the improved cell attachment [47].

\section{Cell penetration}

Cell penetration experiment was designed to culture the mouse fibroblast line NIH 3T3 cells on the surface of 


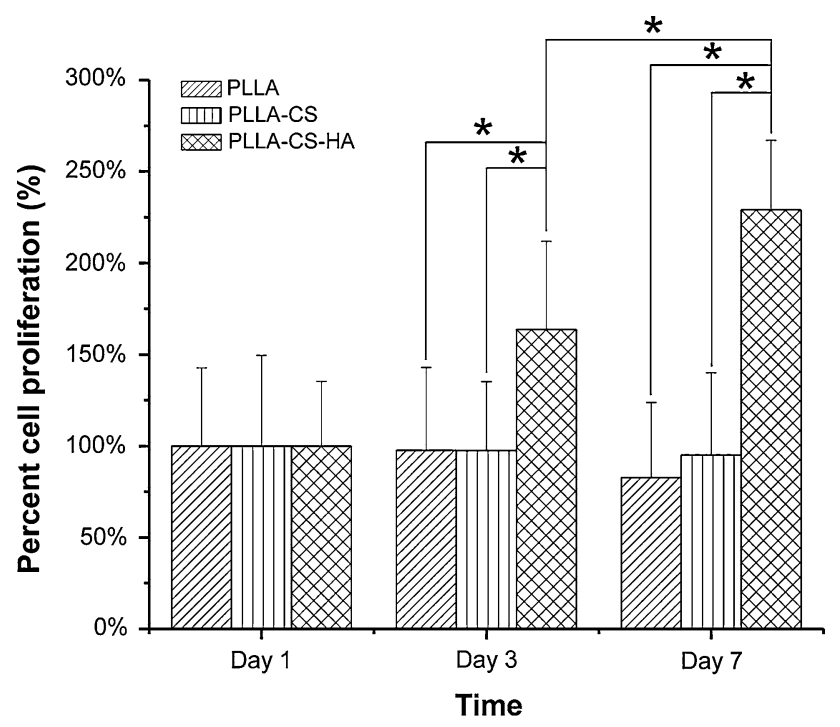

Fig. 7 Cell proliferation. MC3T3 E1 proliferation on PLLA, PLLACS, and PLLA-CS-HA membranes after 1, 3, and 7 days of cell culture $(* P<0.05, n=30)$

PLLA side of the PLLA-CS membrane. The nuclear of fibroblast NIH 3T3 was blue after DAPI staining and visualized under ultraviolet light; the membranes image was taken in bright field. Figure 8 is a merged image of both optical and fluorescence micrograph images. After being cultured on one side of the membrane for 5 days, the NIH 3 T3 cells remained growing on the top of the membrane and some penetrated into the membrane, but could not pass through.

The invasion of the fibroblast tissue is a large obstacle to periodontal tissue regeneration due to the huge difference in the growth rate. This question is quite prevalent in periodontal membrane implanting but has not got enough

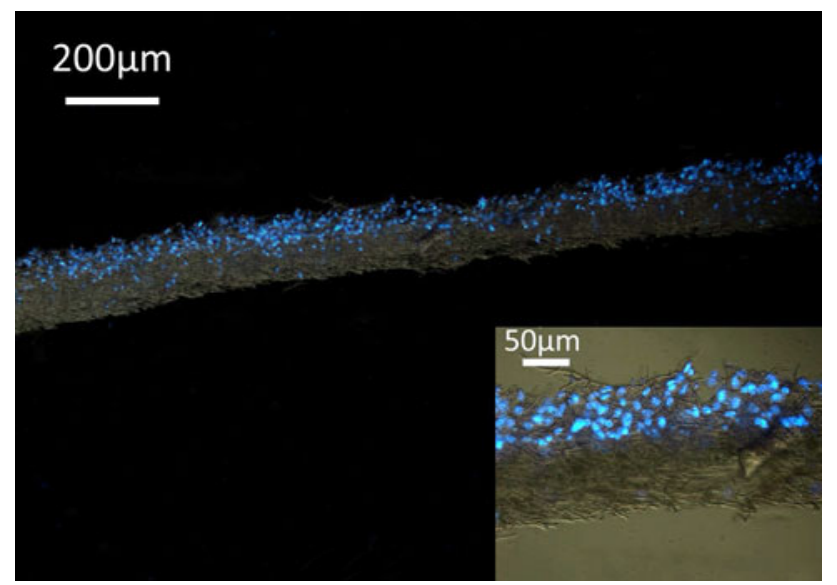

Fig. 8 Cell penetration. Optical and fluorescence merged image of PLLA-CS membrane's longitudinal section (inset showing a higher magnification). Fibroblast NIH 3T3 was cultured on one side of PLLA-CS membrane for 5 days and remained on top of the electrospun membrane attention. To address this question, electrospinning technique was used to fabricate the composite membrane in this study. The pore size is adjustable by fine-tuning the electrospinning conditions to make them small enough to prevent the fibroblast cell from penetrating to the other side of the membrane [48]. The result in Fig. 8 indicated that the porous PLLA-CS membrane (the pore size of the modified PLLA-CS membrane was $2.76 \pm 0.72 \mu \mathrm{m}$ under our experimental condition) was able to prevent the fibroblast from penetrating the membrane and could prevent the epithelial and connective tissue down-growth into the defect in periodontitis treatment. This novel design of periodontal-guided regeneration membrane has not been reported elsewhere.

\section{Conclusion}

Biodegradable electrospun PLLA/chitosan membrane has been successfully prepared by aminolysis method. The modification of chitosan promotes the hydrophilicity, enhances the bioactivity, and accelerates the degradation rate of PLLA electrospun membrane. In vitro cell culture shows that the modified membrane has a better biocompatibility and promotes cell proliferation compared with pure PLLA. Besides, it can also prevent fibroblasts invasion. Therefore, biodegradable electrospun PLLA/chitosan membrane have greater opportunity to be used as guided regeneration membrane for treating periodontitis.

Acknowledgements We appreciate the support from the National Natural Science Foundation of China (Grants 30970727 and 31070844).

Open Access This article is distributed under the terms of the Creative Commons Attribution License which permits any use, distribution, and reproduction in any medium, provided the original author(s) and the source are credited.

\section{References}

1. Pihlstrom BL, Michalowicz BS, Johnson NW (2005) Lancet 366(9499):1809

2. Paoletti R, Gotto AM, Hajjar DP (2004) Circulation 109(23 Suppl 1):III20

3. Taylor GW (2001) Ann Periodontol 6(1):99

4. Kiran M, Arpak N, Unsal E, Erdoğan MF (2005) J Clin Periodontol 32(3):266

5. Grossi SG, Skrepcinski FB, DeCaro T, Robertson DC, Ho AW, Dunford RG, Genco RJ (1997) J Periodontol 68(8):713

6. Skaleric U, Schara R, Medvescek M, Hanlon A, Doherty F, Lessem J (2004) J Int Acad Periodontol 6(4 Suppl):160

7. Dvorak HF, Sioussat TM, Brown LF, Berse B, Nagy JA, Sotrel A, Manseau EJ, Van de Water L, Senger DR (1991) J Exp Med 174(5): 1275 
8. Langer R, Vacanti J (1993) Science 260(5110):920

9. Yamada Y, Ueda M, Hibi H, Baba S (2006) Int J Periodontics Restorative Dent 26(4):363

10. Nyman S, Gottlow J, Karring T, Lindhe J (1982) J Clin Periodontol 9(3):257

11. Nyman S, Gottlow J, Lindhe J, Karring T, Wennstrom J (1987) J Periodontal Res 22(3):252

12. Polimeni G, Koo K-T, Pringle GA, Agelan A, Safadi FF, Wikesjö UME (2008) Clin Implant Dent Relat Res 10(2):99

13. Magnusson I, Batich C, Collins BR (1988) J Periodontol 59(1):1

14. Villar CC, Cochran DL (2010) Dent Clin North Am 54(1):73

15. Simion M, Baldoni M, Rossi P, Zaffe D (1994) Int J Periodontics Restorative Dent 14(2):166

16. Rothamel D, Schwarz F, Sager M, Herten M, Sculean A, Becker J (2005) Clin Oral Implant Res 16(3):369

17. Yan S, Xiaoqiang L, Lianjiang T, Chen H, Xiumei M (2009) Polymer 50(17):4212

18. Athanasiou K (1996) Biomaterials 17(2):93

19. Trejo PM, Weltman R, Caffesse RG (1998) J Periodontol 69(1): 14

20. Kosher RA, Lash JW, Minor RR (1973) Dev Biol 35(2):210

21. Thein-Han WW, Kitiyanant Y (2007) J Biomed Mater Res B Appl Biomater 80(1):92

22. Cui W, Cheng L, Li H, Zhou Y, Zhang Y, Chang J (2012) Polymer 53(11):2298

23. Amaral IF, Granja PL, Barbosa MA (2005) J Biomater Sci Polym Ed 16(12): 1575

24. Lindberg B, Maripuu R, Siegbahn K, Larsson R, Gölander C-G, Eriksson J (1983) J Colloid Interface Sci 95(2):308

25. Khang G, Choee J-H, Rhee JM, Lee HB (2002) J Appl Polym Sci $85(6): 1253$

26. Jung B-O, Kim C-H, Choi K-S, Lee YM, Kim J-J (1999) J Appl Polym Sci 72(13):1713

27. Thanou M, Verhoef JC, Junginger HE (2001) Adv Drug Deliv Rev 52(2):117
28. Ono K, Saito Y, Yura H, Ishikawa K, Kurita A, Akaike T, Ishihara M (2000) J Biomed Mater Res 49(2):289

29. Zhu Y, Gao C, He T, Shen J (2004) Biomaterials 25(3):423

30. Liu Y, He T, Gao C (2005) Colloids Surf B Biointerfaces 46(2):117

31. Dias CI, Mano JF, Alves NM (2008) J Mater Chem 18(21):2493

32. Varma H (1999) Biomaterials 20(9):879

33. Linde A (1995) Connect Tissue Res 33(1-3):163

34. Tanahashi M, Matsuda T (1997) J Biomed Mater Res 34(3):305

35. Tanaka S, Shiba N, Senna M (2006) Sci Technol Adv Mater $7(2): 226$

36. Li J, Chen Y, Yin Y, Yao F, Yao K (2007) Biomaterials 28(5):781

37. Middleton JC, Tipton AJ (2000) Biomaterials 21(23):2335

38. Kim YH, Park JH, Lee M, Kim Y-H, Park TG, Kim SW (2005) J Control Release 103(1):209

39. Cui W, Li X, Chen J, Zhou S, Weng J (2008) Cryst Growth Des $8(12): 4576$

40. Cui W, Li X, Zhou S, Weng J (2008) Polym Degrad Stab 93(3):731

41. Zhang X, Hua H, Shen X, Yang Q (2007) Polymer 48(4): 1005

42. Bottino MC, Thomas V, Schmidt G, Vohra YK, Chu T-MG, Kowolik MJ, Janowski GM (2012) Dent Mater 28(7):703

43. Tjia JS, Aneskievich BJ, Moghe PV (1999) Biomaterials 20(23-24):2223

44. Lao L, Tan H, Wang Y, Gao C (2008) Colloids Surf B Biointerfaces $66(2): 218$

45. Sakaguchi T, Horikoshi T, Nakajima A (1981) Agric Biol Chem 45(10): 2191

46. Cui W, Li X, Xie C, Chen J, Zou J, Zhou S, Weng J (2010) Polymer 51(11):2320

47. Sill TJ, von Recum HA (2008) Biomaterials 29(13):1989

48. Li W-J, Laurencin CT, Caterson EJ, Tuan RS, Ko FK (2002) J Biomed Mater Res 60(4):613 